\title{
Dynamic monitoring of a bi-enzymatic reaction at a single biomimetic giant vesicle
}

Received 00th January 20xx, Accepted 00th January 20xx DOI: $10.1039 / x 0 x \times 00000 x$

\author{
Pauline Lefrançois ${ }^{a}$, Bertrand Goudeau ${ }^{a}$, Stéphane Arbault* ${ }^{a}$
}

\begin{abstract}
Giant unilamellar vesicles were used as individual biomimetic micro-reactors wherein a model bi-enzymatic reaction involving a glucose oxidase (GOx) and the horseradish peroxidase (HRP) was monitored by confocal microscopy. These giant vesicles were formed from a natural mix of phospholipids in physiological conditions of $\mathrm{pH}$ and osmolarity (phosphate buffer, pH 7.4, 330 mOsm). The so-called Amplex Red assay, which generates the highly fluorescent resorufin species, was performed in individual vesicles and used to report on the progress of the whole reaction. We aimed at controlling kinetically and quantitatively the different steps of the bi-enzymatic reaction in vesicles. To do so, substrates (glucose and Amplex Red) were provided in individual reactors by two ways. Electro-microinjection allowed to control volume variations owing to a reservoir of lipids connected to the vesicle membrane. Alternatively, substrates could passively diffuse from the surrounding medium to the vesicle compartment. The semi-permeability feature of the phospholipidic membrane was characterized for all substrates and products while we demonstrated that enzymes remain sequestrated in the vesicles after their injection. The Amplex Red assay was thus achieved in individual vesicles under steady-state conditions, and could pursue over tens of minutes. Such giant vesicles are stable, fully compatible with media used for bioanalyses and allow out-of-equilibrium reactions at time-scales compatible with living reaction dynamics, making them a good choice for the development of minimal cell-like systems.
\end{abstract}

\section{Introduction}

Liposomes are widely used as bio-compatible carriers ${ }^{1}$, as well as for studying membrane biophysical properties ${ }^{2}$ or biochemical reactions ${ }^{3}$. Their membrane can be composed of various synthetic or natural phospholipids and fatty acids ${ }^{4}$, mimicking the basic structure of living cell membranes. The size of vesicles (small, large, giant) and the number of their bilayers can be adjusted depending on the preparation technique $^{2,5}$ (sonication ${ }^{6}$, extrusion ${ }^{7}$, freeze-thawing, electroformation $^{8-11}$, rehydration ${ }^{12-14}$, microfluidic ${ }^{15,16}$, etc.). Phospholipid unilamellar vesicles are used to perform diverse biochemical and biological reactions ${ }^{17}$. However, when compared to biological cells, these synthetic systems often lack a transport of reactants and products between the inside of vesicles and their external environment. Consequences are that reaction dynamics cannot be studied due to a fast runout of encapsulated substrates (contained in nanolitre volumes), or when reactions stop when reaching their thermodynamic equilibrium $^{18}$. To overcome this major limitation and reach

a. Univ. Bordeaux, CNRS, Bordeaux INP, ISM, UMR 5255, F-33400, Talence, France. + Corresponding author: Dr. Stéphane ARBAULT; email: stephane.arbault@ubordeaux.fr; Tel: +33556847907 .

Electronic Supplementary Information (ESI) available: Material and methods, Reactional pathways, Bulk kinetic study by fluorescence spectroscopy, Response as function of Amplex Red concentration, kinetic analyses of fluorescence decays, tables giving the detailed parameters for each experiment in Figures, Lipid composition. See DOI: 10.1039/x0xx00000x steady-state activities inside these reactors, a continuous transport of substrate(s) from the outside to the inside of the compartment is required. This can be facilitated by membrane proteins $^{19}$ such as pore-forming toxins ( $\alpha$-hemolysin) ${ }^{15,20}$ or peptides (melittin) ${ }^{21}$, which allow passive transport of molecules up to $\sim 5 \mathrm{~nm}$ in diameter. Alternatively, semipermeable membranes allow the selective diffusion of certain molecules (typically small molecules below $200 \mathrm{~g} \cdot \mathrm{mol}^{-1}$ molecular weight, or $1 \mathrm{~nm}$ in diameter) while larger ones (diameter $>1 \mathrm{~nm}$, e.g. enzymes or nucleic acids) remain entrapped in the micro-reactor. Giant unilamellar vesicles (GUVs: 10-200 $\mu \mathrm{m}$ diameter) have raised growing interest as biomimetic reactors, owing to their size, compatibility with proteins and with usual biochemical aqueous buffers, a control of passive diffusion across their membrane and ease for in situ analytical monitoring, particularly by optical microscopy. Herein, we report the preparation of GUVs from a natural phospholipid mix, with semi-permeable membrane properties and use them to monitor a bi-enzymatic reaction in real-time and in individual vesicles. GUVs were formed by a dehydrationrehydration method ${ }^{12}$. With this technique, GUVs grow on lipid reservoirs. The connection between the membrane and the reservoir allows the transfer of lipids from one to the other. Consequently, the GUV membrane surface can increase or decrease as a function of internal volume changes during microinjection or subsequent to osmotic changes. Enzymatic reactants were either directly encapsulated during GUVs growth when they were solubilized in the rehydration solution, 
or microinjected inside vesicles to trigger the reactions. The first approach allows the incorporation of species at a fixed concentration inside all the GUVs whereas the second enables the encapsulation of defined volumes in single vesicles. These were used to achieve a model bi-enzymatic reaction coupling the glucose oxidase (GOx) and horseradish peroxidase (HRP) reactions. Reaction dynamics were monitored by confocal microscopy using the fluorescent Amplex Red assay. The assay was conducted systematically by incorporating each reactant in vesicles using passive diffusion and microinjection. We monitored GOx and HRP activities in single GUVs under steadystate condition for up to $30 \mathrm{~min}$. Our study shows how simple liposomes can be used as bioreactors by combining diffusion and electro-microinjection, paving the way for more complex enzymatic pathways to mimic out-of-equilibrium cell-like activities.

\section{Experimental}

Chemicals. Soybean polar extract (Avanti Polar Lipids, reference 541602) was solubilized in chloroform (Sigma FR) According to the supplier, the lipid composition of the soybean polar extract is $45.7 \mathrm{wt} / \mathrm{wt} \%$ phosphatidylcholine, $22.1 \mathrm{wt} / \mathrm{wt} \%$ phosphatidylethanolamine, $18.4 \mathrm{wt} / \mathrm{wt} \%$ phosphatidylinositol, $6.9 \mathrm{wt} / \mathrm{wt} \%$ phosphatidic acid and $6.9 \mathrm{wt} / \mathrm{wt} \%$ unknown. Preformed vesicles were prepared in KPi buffer (see next section) composed of $5 \mathrm{mM}$ TrizmaBase (Sigma FR), $30 \mathrm{mM}$ $\mathrm{K}_{3} \mathrm{PO}_{4}$ (ABCR), $30 \mathrm{mM} \mathrm{KH}{ }_{2} \mathrm{PO}_{4}$ (Acros Organics), $1 \mathrm{mM} \mathrm{MgSO}$, $7 \mathrm{H}_{2} \mathrm{O}$ (Sigma $\mathrm{FR}$ ), $0.5 \mathrm{mM}$ EDTA (Sigma FR) and $\mathrm{pH}$ was adjusted at 7.4 by addition of $\mathrm{H}_{3} \mathrm{PO}_{4} 98 \%$.

All other experiments were conducted in phosphate-buffered saline solution (10 mM phosphates, $2.7 \mathrm{mM} \mathrm{KCl}, 138 \mathrm{mM} \mathrm{NaCl}$, Sigma FR, reference P5368) prepared by dissolving the aforementioned salts in pure MilliQ water (Millipore, Integral 3, $0.22 \mu \mathrm{m}$ MilliPak filter). Hydrogen peroxide (Fluka Analytics), glucose (Sigma FR), horseradish peroxidase (ThermoFisher, A22188 kit) and glucose oxidase from Aspergillus Niger (Sigma $\mathrm{FR}$, reference $\mathrm{G7141}, 10 \mathrm{kU}$ ) were solubilized and diluted in PBS at the desired concentrations. Amplex Red (ThermoFisher, A22188 kit) was freshly solubilized in DMSO and then diluted in PBS prior to each experiment.

Preparation of the giant unilamellar vesicles in physiological buffer. A detailed protocol for giant unilamellar vesicles has been described elsewhere and has been adapted from a dehydration-rehydration technique originally developed by Criado and Keller ${ }^{14}$. Briefly, a natural extract from soybean (see the Chemicals section) was solubilized in pure chloroform at 10 $\mathrm{mg} \cdot \mathrm{mL}^{-1}$ in a $10 \mathrm{~mL}$ pear-shaped flask. The solvent was evaporated with a rotary evaporator during 3 to $5 \mathrm{~h}$ (80-100 rpm) leading to the formation of a lipid film. KPi buffer (see composition in the Chemicals section) was added in the flask allowing a gentle hydration of the film ( $\sim 12 \mathrm{~h}$ at $\left.4^{\circ} \mathrm{C}\right)$. Glycerol was then added to the suspension (10 vol\%). Finally, a sonication step allowed the formation of SUV-MLVs (sonication bath, $3 \mathrm{~min}$ ).

GUVs were obtained from this preformed liposome suspension by a second dehydration-rehydration step. $5 \mu \mathrm{L}$ of the suspension were dropped onto a glass coverslip and dehydrated for $30 \mathrm{~min}$ in a vacuum desiccator using a vacuum membrane pump. The dehydrated lipids form a bevelled-edge transparent film with salt crystals in the centre. The presence of glycerol prevents from complete dehydration of the lipids. Final rehydration was obtained by addition of PBS $(0.5 \mathrm{~mL})$ onto the coverslip. Osmotic gradient allows the swelling of GUVs on lipid reservoirs. After 10-15 min of equilibrium, the vesicles suspension was transferred onto coated glass slides (see next section). After $\sim 10 \mathrm{~min}$, vesicles had sedimented and were immobilized on the slide by interaction with the coating material (see next section).

Preparation of microscopy slides. Glass slides were thoroughly cleaned with ethanol and milliQ water and dried under pressurized air flow. Surface activation was conducted with an oxygen plasma (PDC-002, Harrick Plasma, USA) for $10 \mathrm{~min}$. SU8-2002 epoxy photoresist (Micro-chem) was then spin coated on the slides (100 rpm $\cdot \mathrm{s}^{-1} \mathrm{ramp}$ up to $1000 \mathrm{rpm}$, then kept at this speed during $1 \mathrm{~min}$ ). The photoresist was prebaked at $95^{\circ} \mathrm{C}$ for $5 \mathrm{~min}$ on a hotplate, UV exposed at 40 $\mathrm{mW} \cdot \mathrm{cm}^{-2}$ during $8 \mathrm{~s}$ (Kloe, UV Kub) and post-baked at $95^{\circ} \mathrm{C}$ during $5 \mathrm{~min}$ on a hotplate. The SU8 substrate was chosen because it had already been used in similar experiments. It was shown to present a slight interaction with phospholipids which is sufficient to immobilize GUVs, without deforming or damaging the membrane ${ }^{12}$. The coated film thickness is estimated at $\sim 3 \mu \mathrm{m}$ according to supplier's tables and spin speed used here.

Microscopy observations. All images were obtained on an inverted laser scanning confocal microscope (Leica TCS-SP5 II) with a 20x dry objective (HCX PL Fluotar L, IP1, NA 0.4). We used an argon laser (output power 20\%) to excite resorufin at $514 \mathrm{~nm}$ (acousto-optic tunable filter, 30\%). Emission was collected within the adjusted wavelength window of 550 to $710 \mathrm{~nm}$. Detection was done with a photomultiplier set to a gain of $800 \mathrm{~V}$. Experiments were monitored in time-lapse mode with a $1.47 \mathrm{~s}$ time-interval between acquisitions $(700 \mathrm{~Hz}$ frequency, $1024 \times 1024$ pixels window; no averaging nor accumulation).

Electro-microinjection. Microinjection experiments were performed with manual micromanipulators using mechanical and piezoelectric control of translation stages (ThorLabs PCS5200). Vesicles were electro-microinjected with a glass micropipette formed from a glass capillary (WPI, TW100F-4) pulled with a pipette puller (PC-100 model, Narishige, Japan) with parameters preliminary determined to provide pipette tips with a diameter below $1 \mu \mathrm{m}$ (heat level $=82$, force $=2.45$ N). A platinum wire (Good Fellow PT005140) was inserted in the pipette and another one was positioned randomly in the solution used as a counter electrode. Both wires were connected to an electric pulse generator (Digitimer DS2AMkII). When the tip of the pipette was in touch with the vesicle membrane, the application of electric pulses $(40 \mathrm{~V}, 20 \mathrm{~ms})$ allowed the formation of a local pore in the membrane ${ }^{12,22}$ (not observable here). The pipette was moved simultaneously to application of pulses leading to its penetration inside the vesicle. Finally, the solution contained in the pipette was 
injected with an air injector (Eppendorf FemtoJet). One should note that this type of injectors doesn't allow the injection of a pre-set volume, but instead the injection is based on an injection pressure and duration. Pulling pipettes present variability in their tip opening size (Figure S1), thus variations of solution flow during injection for a specific pressure and injection duration were observed. Consequently, the injected volumes and therefore the quantities of injected reactants were systematically calculated based on the GUV diameter and inner volume increases after each injection and for each experiment.

Image analyses. All images were analysed using the software Image $^{23}$. For the cross-section profile analysis, a line-type region of interest (ROI) was drawn with a thickness of $18.3 \mu \mathrm{m}$ across a GUV. For time lapse experiments, circular ROls were drawn to analyse the fluorescence at the equatorial plane of the microinjected GUVs. Each experiment was reproduced on at least ten different GUVs (not necessarily from the same sample). The fluorescence intensity displayed on the figures corresponds to the raw data without further processing, whereas time offsets were applied to set the start of the experiment at $t=0$. Each vesicle and each micropipette being different from one another (mostly in size and tip opening diameter respectively), the reported fluorescence intensity varied between replicates but the trend remained the same for all replicates of a specific experiment. We therefore show the analysis of only 1 GUV per experiment, representing the general observed behaviour for each condition.

In most of the results, fluorescence decays are observed after the injection of resorufin or after the onset of the enzymatic reactions. To assess for the effect on fluorescence variations of resorufin photobleaching versus its diffusion from the vesicle to the outer solution, all decays were fitted with one and two exponential decay curves. Results of treatments for each curve are reported in Supplementary Materials (Figures S7 to S10).

\section{Results and discussion}

We studied at the level of single GUVs a bi-enzymatic reaction involving a glucose oxidase (GOx, $160 \mathrm{kDa}$ dimer) and the horseradish peroxidase (HRP, $40 \mathrm{kDa}$ ). The first enzyme catalyses the oxidation of $\beta$-D-glucose to D-glucono- $\delta$-lactone with a production of hydrogen peroxide $\left(\mathrm{H}_{2} \mathrm{O}_{2}\right)$. This is a well characterized reaction ${ }^{24,25}$, used herein as a model. Then, hydrogen peroxide serves as a substrate to HRP, which oxidizes Amplex Red (AR; $\mathrm{N}$-acetyl-3,7-dihydroxyphenoxazine), to form the highly fluorescent species resorufin ${ }^{26}$ (Figure 1 ). The AR assay is commonly used in bioanalytical techniques to assess hydrogen peroxide released by cells and biochemical activities due to oxidases ${ }^{27}$. Also, it was used to monitor enzymatic reactions in micro and nano-volumes ${ }^{28}$. However, even though the AR assay is considered as a routine technique, it has been demonstrated that precautions should be taken to ensure accurate data interpretations ${ }^{29,30}$. The main recommendation is to avoid high concentrations of hydrogen peroxide. Above $100 \mu \mathrm{M} \mathrm{H}_{2} \mathrm{O}_{2}$, enzyme inactivation (HRP) or substrate inhibition (Amplex Red) are described ${ }^{29}$. Thus, we did a preliminary bulk spectroscopic study, which provided us with the optimal conditions to get the fastest kinetics of the bienzymatic system (Figures S2, S3 and S4) and be able to later observe a dynamic fluorescence report of the AR assay in GUVs.

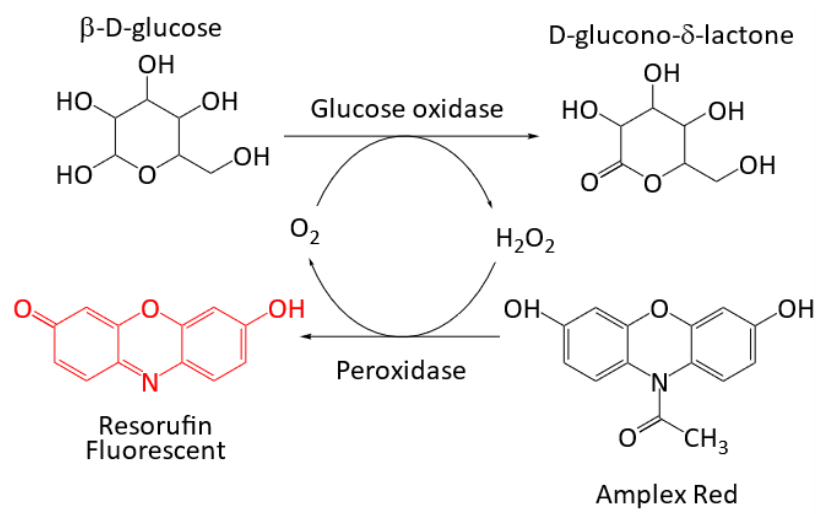

Figure 1. Scheme of the bienzymatic reaction used in this study. $\beta$-D-glucose is catalytically oxidized via glucose oxidase $(\mathrm{GOx})$ to $\mathrm{D}$-glucono- $\delta$-lactone and hydrogen peroxide. Hydrogen peroxide then oxidizes the fluorogenic compound Amplex Red, catalysed by the horseradish peroxidase (HRP), to produce the fluorescent final product resorufin.

The Amplex Red assay was conducted in single vesicles following a step by step method. Each result presented below is the typical trend obtained for each experiment. Every experiment was repeated at least with ten vesicles. Trends were very reproducible at each step although we observe differences in the fluorescence intensity amplitudes between different experiments. This is explained by the differences of size between each GUV and each micropipette, resulting in various reactant final concentrations inside a GUV. As function of these individual parameters, convection and mixing times after injections might therefore vary slightly before reaching steady-state. Such variability is inherent to any single vesicle study with a population poly-dispersity.

The first tested compound was the end product of the assay, resorufin. Preformed vesicles were dehydrated and rehydrated with PBS (10 mM, pH 7.4) resulting in the formation of a GUV connected to a lipid reservoir (bright spots on Figure 2A). A GUV was then microinjected with a resorufin solution (Figure 2). The cross-section fluorescence profile obtained by laser scanning confocal microscopy (LSCM) at the equatorial plane of the vesicle (Figure $2 \mathrm{~A}$ and $\mathrm{B}$ ), shows a maximal intensity at its centre, a sharp decay at the membrane, due to the partition coefficient of the membrane for resorufin, and a fluorescent halo surrounding the GUV due to the molecule diffusion toward the outside. 

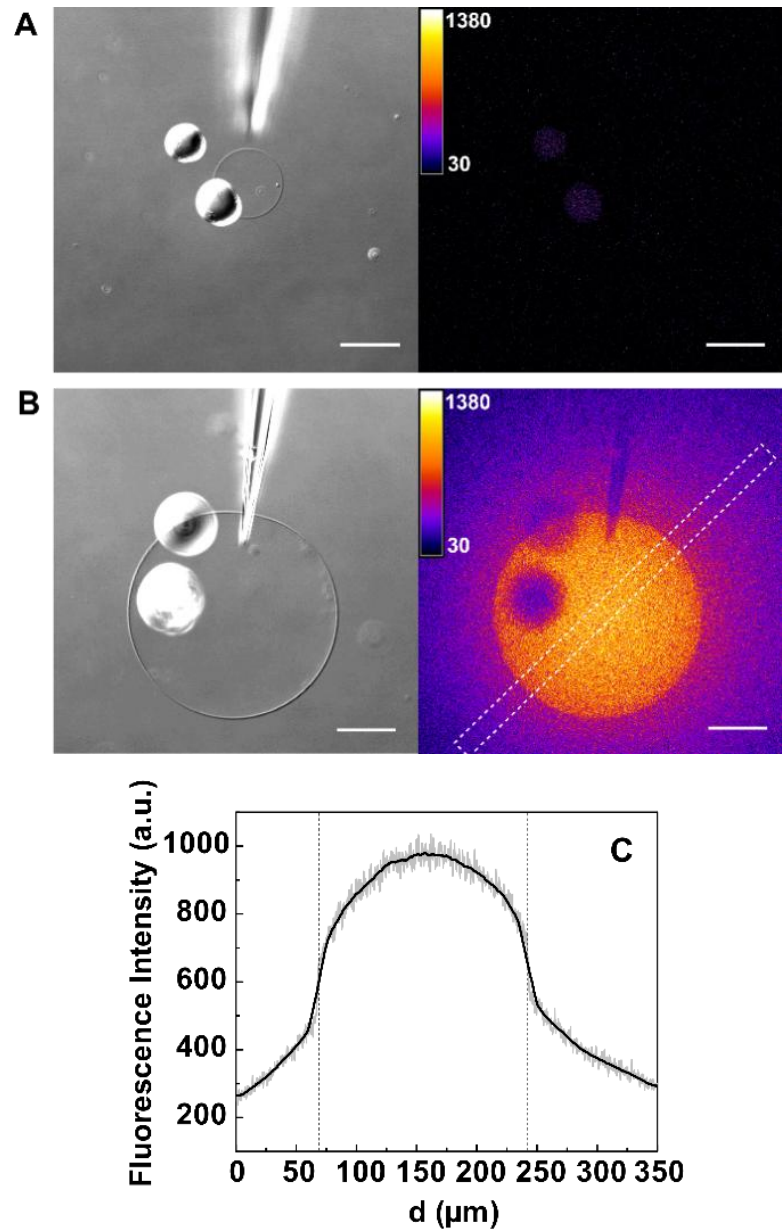

Figure 2. Micro-injection of resorufin in a single GUV monitored by LSCM. A Microscopy images of a GUV (connected to its lipid reservoir) before injection. B. Same GUV during injection of resorufin (injection duration $=30 \mathrm{~s}$ ). Left: differential interference contrast image; Right: emitted fluorescence. Scale bar: $50 \mu \mathrm{m}$. Pseudocolour, lookup table (LUT): fire. The dashed zone corresponds to the region of interest used to plot the cross-section profile in C. C. Fluorescence profile across the GUV micro-injected with resorufin. The black trace is the 50-point moving average of the grey trace.

Figure 3 depicts the variations of the fluorescence intensity inside a GUV upon sequential micro-injections of a resorufin solution (initial concentration of $2.5 \mu \mathrm{M}$ in the pipette). The final concentration of resorufin in the GUV is calculated as 0.24 $\pm 0.08 \mu \mathrm{M}$ (details in table S1, ESI). We observed a fast increase of the fluorescence right after the injection, followed by its rather fast decrease immediately after the end of injection. Each time, the signal reached back the background value in $\sim 1 \mathrm{~min}$, driven by the concentration gradient between the outside and the inside of the vesicle (inner volume: $2-3 \mathrm{~nL}$; outer volume: $500 \mu \mathrm{L}$ ). This experiment was repeated at least 3 times per vesicle, and for several tens of them. We always obtained the same profiles.

The fluorescence decays may be attributed to the simple diffusion of resorufin and/or its photobleaching due to laser exposure (see experimental section). The effect of photobleaching was shown as minimal based on two results: 1 . when a solution of resorufin (up to $50 \mu \mathrm{M}$ in PBS), even in the presence of GUVs (Figure S5), was exposed under the same experimental conditions as the above, the local decrease of fluorescence intensity was weak (below $5 \%$. over a $1 \mathrm{~min}$ timescale); 2. the fluorescence decays were fitted with exponential decay functions (one or two components; see Figure 57) to assess for the effect of photobleaching. In this case, and in further experiments, a very slow decay function could possibly be attributed to the dye photobleaching, though a diffusion kinetic was mainly observed. Moreover, the flux of resorufin provided by the pipette in the GUV led, after each injection, to an ensuing fluorescence in the solution surrounding the GUV (as shown also on Figure 2), which shows that resorufin diffuses within a second time-scale across the membrane of these giant liposomes. Resorufin permeation is indeed reported as dependent on membrane compositions (phospholipids, sterols, proteins) of vesicles, interfaces or cells $^{28,31}$.

At this initial stage of the study, several control experiments were made to check whether the microinjection and electrical pulses may induce damages to the membrane resulting in the formation of pores and an increased permeability. GUVs were prepared in PBS followed by an addition of resorufin in their outer solution. In less than 30 seconds, all GUV lumina were filled with resorufin (Figure S5). The same experiment was also performed with fluorescein and calcein fluorophores; both are anionic at $\mathrm{pH} 7.4$ but calcein is a slightly larger molecule than fluorescein (6.5 $\AA$ Stocks' radius for calcein ${ }^{32}$ and $\sim 5 \AA$ for fluorescein ${ }^{33}$ ) (Figure S5). A similar filling of GUVs was observed within less than a minute for both molecules. This demonstrates that the semi-permeable feature is intrinsic to the GUVs, according to their preparation method and composition, and is not influenced by electric pulses, nor to membrane tension due to microinjection.

In order to evaluate if the permeability would be associated with the presence of transient pores, we performed a negative contrast experiment, similar than before, with a much larger molecule, a Dextran-FITC (70 kDa; Stocks' radius $6.0 \mathrm{~nm}^{34}$ ). A progressive diffusion of Dextran-FITC toward the vesicle interior was also observed, though at a very slow rate (several hours), notably compared to the size of the vesicles. One should note as well that Dextran polymers are coil-shaped (length $44 \mathrm{~nm}$, width 2.5 to $3 \mathrm{~nm}^{35}$ ) and are known to follow reptation movements ${ }^{35}$, transient unfolding and slow diffusion of Dextran chains through small pores in membranes.

These results indicate that the membrane of GUVs is semipermeable essentially to small hydrophilic molecules, including resorufin, calcein and fluorescein. Moreover, the diffusion of these fluorophores across the membrane is homogeneously distributed, either around GUVs after internal microinjections (Figure 2B), or inside their lumen during negative contrast experiments (Figures S5 and S6). This shows that no specific defects at the interface between the vesicle and the lipid reservoir induce any leakage of the vesicle content during the following experiments. 
A

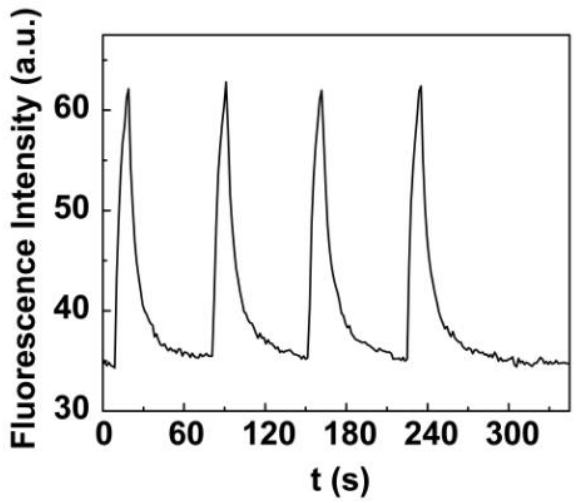

B

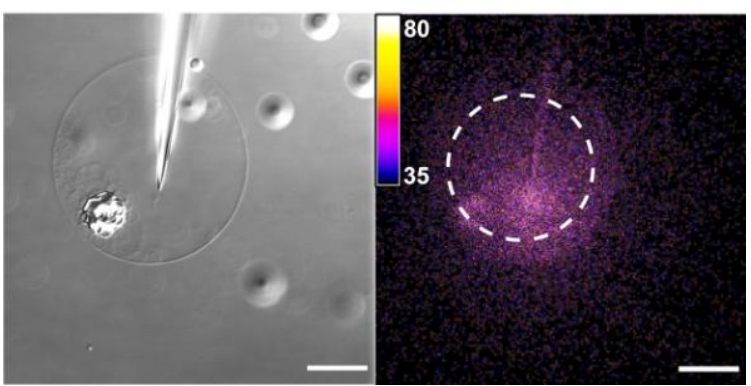

Figure 3. A. Fluorescence intensity variations over time inside a GUV (corresponding to the indicated region of interest) injected 4 times with resorufin B. Microscopy images of a GUV connected to its lipid reservoir during injection of resorufin. Left: differential interference contrast image; Right: emitted fluorescence. Pseudo-colour, LUT: fire. Scale bar: $50 \mu \mathrm{m}$. The region of interest (dashed circle) used for the analysis of the radial fluorescence inside the GUV is delimited by a white dashed line. Injected volumes were estimated by size changes of the GUV to $\sim 240 \pm 50 \mathrm{pL}$ per injection. Injection duration: $5 \mathrm{~s}$. Initial concentration in the pipette: $2.5 \mu \mathrm{M}$; final concentration in GUV $=0.24 \pm 0.08 \mu \mathrm{M}$. Initial GUV diameter: $155 \mu \mathrm{m}$; GUV diameter after 4 injections: $177 \mu \mathrm{m}$.

The AR reaction was then performed in single GUVs. Vesicles were grown on lipid reservoirs by rehydration in PBS supplemented with $10 \mu \mathrm{M} \mathrm{H}_{2} \mathrm{O}_{2}$ (the reaction substrate, see Figure 1 bottom part). Hydrogen peroxide was thus present in the inside and outside of the liposomes, and was prone to diffuse passively across the membrane, as previously reported ${ }^{36}$. We injected a mix of the co-reactant AR $(6.8 \pm 3.2$ $\mu \mathrm{M}$ final) and HRP enzyme (0.03 $\pm 0.01 \mathrm{U} \cdot \mathrm{mL}^{-1}$ final) in single GUVs. The local fluorescence signal evolution was followed by LSCM (Figure 4). We observed a fast fluorescence increase subsequent to the injection. After injection was stopped, the signal decreased progressively to reach the background level. Five successive injections were performed in the same vesicle, all showing the same response profile and thus the reproducibility of the response. Similar to Figure 3 , the fluorescence decays observed here were analysed kinetically (Figure S8) and could be attributed mainly to the passive diffusion of resorufin across the membrane. Thus, we may conclude that a short injection (5 s) provides only a little amount of AR that is immediately consumed by the reaction (table S2, ESI). AR may also progressively diffuse outside of the GUV. When missing its substrate, the reaction stops very fast after the injection and since resorufin is not sequestrated, spikes of fluorescence are observed. In this first experimental condition; despite the AR enzymatic reaction lasts at most one minute, it can be performed in individual vesicles with a fine temporal and quantitative control of the injected compounds. Nevertheless, the diffusion of AR, and possibly of HRP, across the membrane need to be better understood (see below).

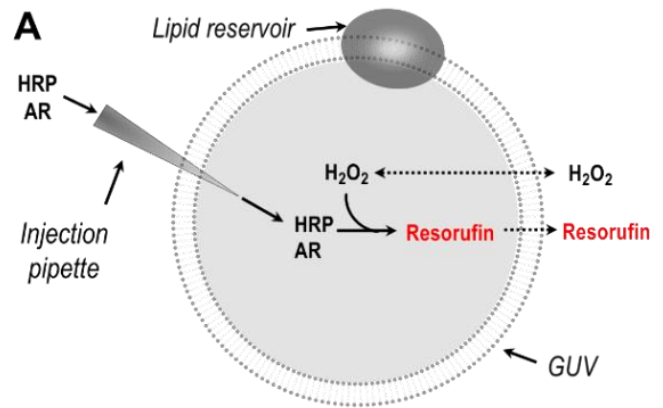

B

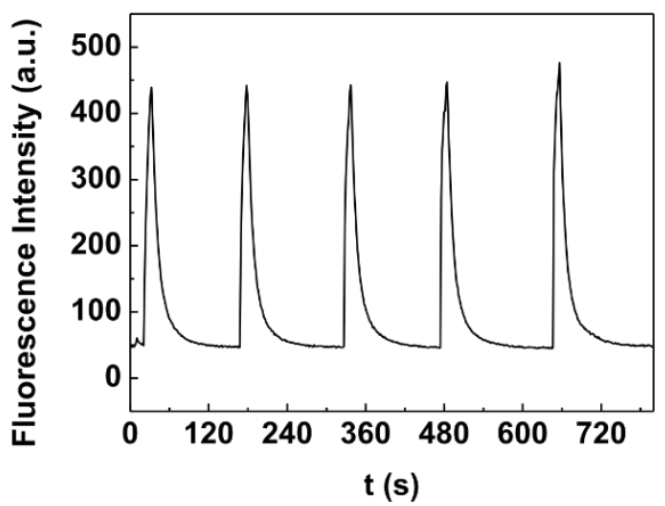

C

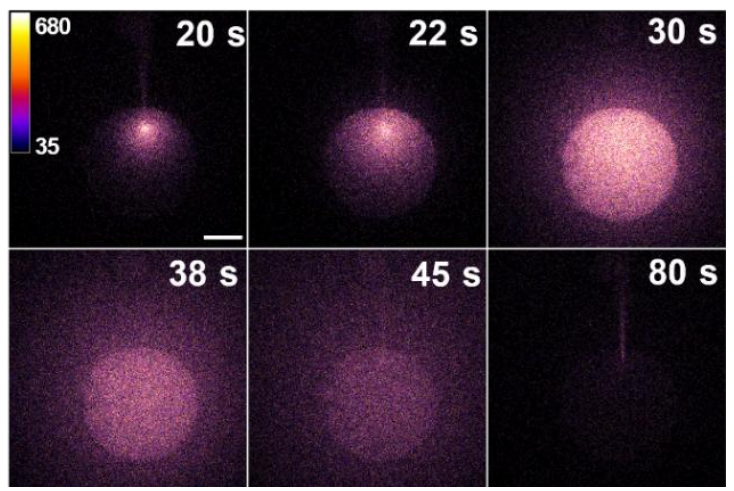

Figure 4. A. Schematic representation of the Amplex Red assay performed in a single GUV. A vesicle containing $\mathrm{H}_{2} \mathrm{O}_{2}(10 \mu \mathrm{M})$ was injected with a mix of $\mathrm{HRP}\left(0.4 \mathrm{U} \cdot \mathrm{mL}^{-1}\right.$ initial concentration) and $\mathrm{AR}(50 \mu \mathrm{M}$ initial concentration). The passive diffusion of $\mathrm{H}_{2} \mathrm{O}_{2}$ is depicted with a dashed double arrow. $\mathrm{B}$. The resorufin production is reported by the mean fluorescence intensity inside the GUV over time detected by LSCM. C. Microscopy time-lapse images obtained by LSCM of a GUV during and after injection, showing the evolution of fluorescence intensity. Scale bar: $50 \mu \mathrm{m}$. Pseudo-colour, LUT: fire. 5 successive injections were performed. The HRP and AR concentrations in the vesicle were calculated for each injection according to the injected volume and averaged for the 5 injections: $0.03 \pm 0.01 \mathrm{U} \cdot \mathrm{mL}^{-1} \mathrm{HRP}$ and $6.8 \pm 3.2 \mu \mathrm{M}$ AR. Average injected volume for each injection $=130 \pm 70 \mathrm{pL}$. Injection duration: $5 \mathrm{~s}$. Initial GUV diameter: $145 \mu \mathrm{m}$; GUV diameter after 5 injections: $160 \mu \mathrm{m}$.

A third experiment was designed to explore this latter point. Vesicles were formed by rehydration in PBS (10 mM, pH 7.4) containing $50 \mu \mathrm{M} \mathrm{H}_{2} \mathrm{O}_{2}$ and $50 \mu \mathrm{M}$ AR. Higher concentrations of both substrates were used in this experiment to maximize their in situ concentrations in vesicles and the observed 
fluorescence response. The conversion reaction started upon a single injection of HRP in a vesicle (Figure 5). Resorufin fluorescence intensity inside the GUV increased, indicating its effective enzymatic production. After $40 \mathrm{~s}$ of injection (corresponding to $270 \mathrm{pL}$ volume; table S3, ESI), the fluorescence kept increasing for about $30 \mathrm{~s}$ and reaches a high intensity level. Then it slowly decreased, over more than 20 min in the representative example displayed here. The whole duration of resorufin production and fluorescence emission was dependent on the GUV dimensions and substrate quantities injected inside.

Overall, this advanced set of experiments demonstrates the achievement of the AR assay in individual GUVs and its timeresolved monitoring. Several interpretations can be considered at this point to explain these results by comparison with Figures 3 and 4 . The decay rate in Figure 5 is $50-100$-fold slower than on Figures 3 and 4. Although diffusion of resorufin toward the external solution continuously occurs as well as a slight photobleaching (kinetic analyses in Figure S9), this result indicates that the production of resorufin inside the GUV is less limited by a substrate runout than previously. Since AR is encapsulated during GUV growth, the concentration inside the vesicle is assumed to be the one of the hydration solution ( 50 $\mu M)$; whereas when the $A R$ is injected together with the enzyme (Figure 4), the average concentration after injection is lower, estimated to $\sim 7 \mu \mathrm{M}$. This 10 -fold difference in concentration changes the reaction rate and allows the reaction to last longer in the case of Figure 5. Moreover, $\mathrm{H}_{2} \mathrm{O}_{2}$ diffuses quickly across the phospholipid membrane and is then continuously provided to the inside of the vesicle. Therefore, if the reaction rate decreases over time due to a substrate runout, it would be most likely because of AR. However, previous studies have shown that $A R$ is able to permeate across phospholipid membranes ${ }^{36}$, while HRP should not. In addition, one may argue that confinement in a vesicle affects the AR assay kinetics because of AR inhibition and/or HRP inactivation, although we optimized the reaction conditions in bulk (Figure S3) and limited hydrogen peroxide concentration to $50 \mu \mathrm{M}$. Therefore, the slow decrease of fluorescence observed on Figure 5, which represents the kinetic slowdown of the AR assay, is due either to a slow diffusion of AR across the membrane, or to a progressive inactivation or inhibition of HRP or AR, respectively. The next study will enable to address this point.
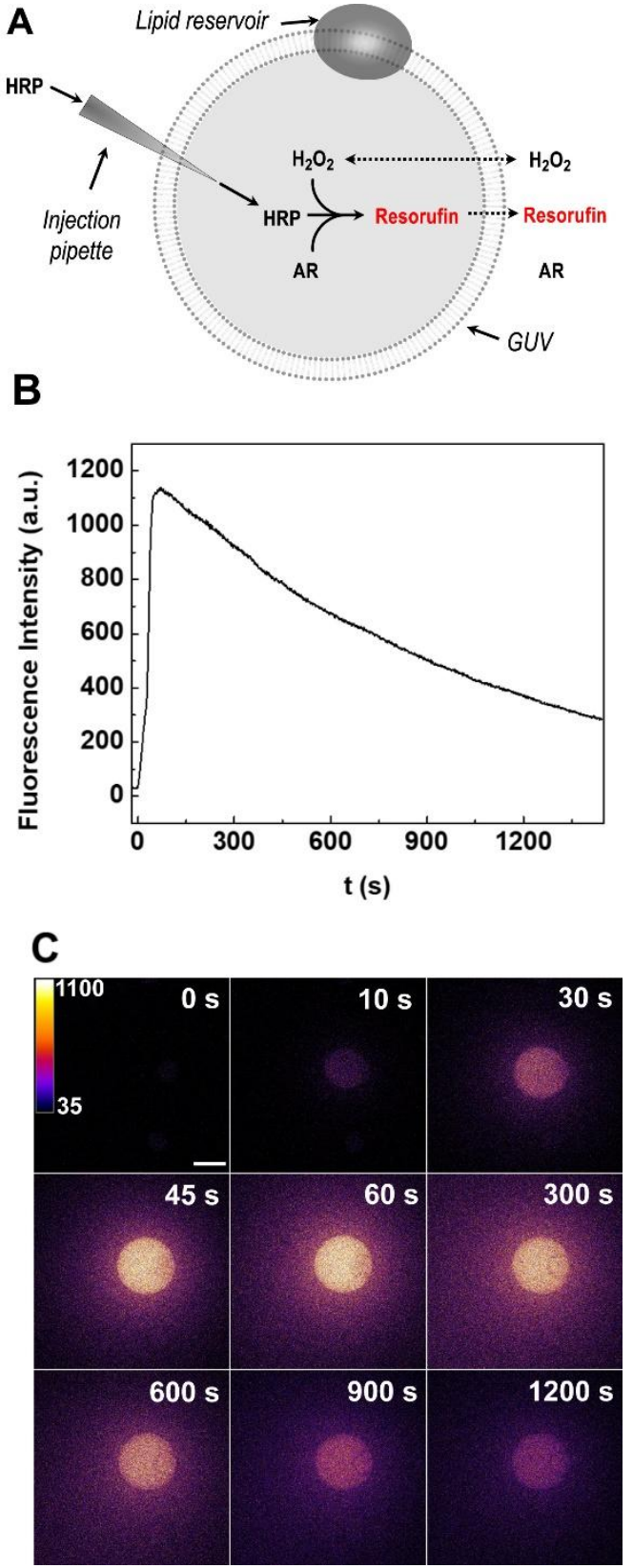

Figure 5. A. Schematic representation of the Amplex Red assay in a single GUV. A hydrogen peroxide $(50 \mu \mathrm{M})$ and AR $(50 \mu \mathrm{M})$-containing GUV was injected once with HRP $\left(0.28 \mathrm{U} \cdot \mathrm{mL}^{-1}\right.$ final). B. The resorufin production is reported by the mean fluorescence intensity inside the GUV over time monitored by LSCM. C. Microscopy time-lapse images obtained by LSCM of a GUV during and after injection, showing the fluorescence intensity increase followed by a slow decay. Scale bar: $50 \mu \mathrm{m}$. Pseudocolour, LUT: fire. Injected volume: $270 \mathrm{pL}$. Initial GUV diameter: $60 \mu \mathrm{m}$; GUV diameter after injection: $90 \mu \mathrm{m}$.

The bi-enzymatic reaction, involving both GOx and HRP, was then performed in GUVs. A glucose solution (100 $\mu \mathrm{M}$ in PBS) was used to rehydrate the lipid film and grow the giant liposomes. The two enzymes (GOx and HRP) were mixed with $A R$ and the solution was subsequently injected inside a vesicle (Figure 6). All concentrations were previously optimized in bulk by spectrophotometry to get a fast resorufin production (Figure S4) and to avoid side perturbations of the AR reaction. We set the GOx reaction condition as the rate limiting step so 
that any produced $\mathrm{H}_{2} \mathrm{O}_{2}$ would be immediately consumed by the AR reaction, avoiding a steady-state oxidative environment. As shown in Figure 6 , successive injections of the -GOX+HRP+AR- cocktail could be performed (details in table S4, ESI), leading each time to a fast increase of the resorufin fluorescence signal inside the GUV, followed by a decrease. After resorufin diffusion to the outside of the vesicle, the final fluorescence intensity reaches the background level (in 1 to 2 min, depending on the vesicle size). As previously, the fluorescence increase is associated to the resorufin production and demonstrates the proper working of the bienzymatic reaction inside the vesicles, which is here limited by AR runout. The fluorescence decrease corresponds to passive diffusion of resorufin across the membrane, as photobleaching was not observed in this case (Figure S10).

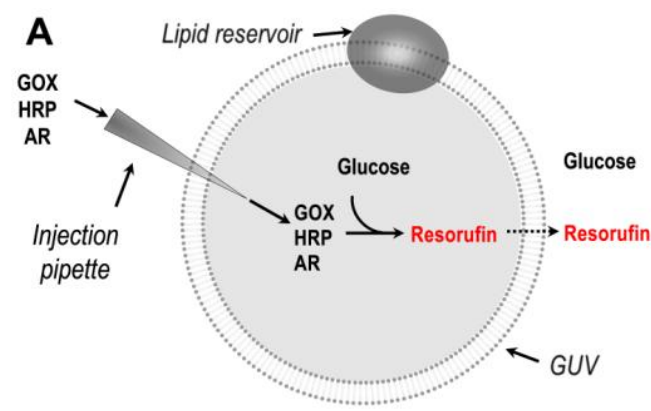

B

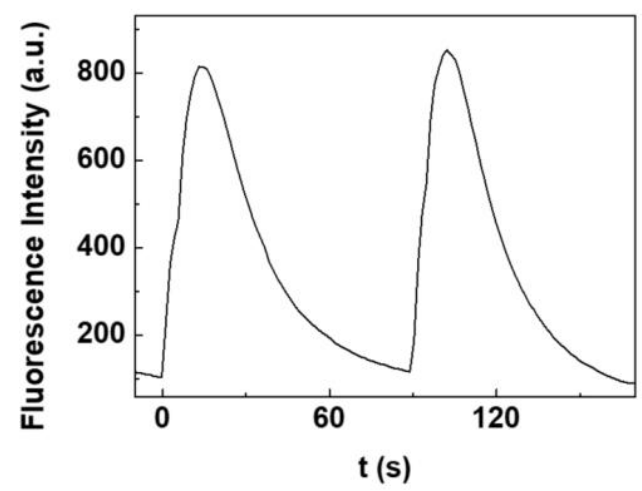

C

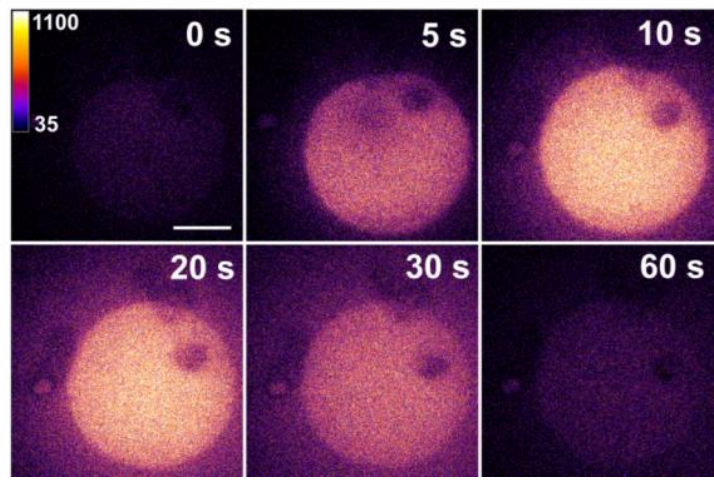

Figure 6. A. Schematic representation of the coupled bi-enzymatic assay in GUVs. A glucose $(100 \mu \mathrm{M})$-containing GUV is injected with a mix of GOx, HRP and AR; in the typical experiment displayed here, their respective concentrations in the vesicle were $0.9 \mathrm{U} \cdot \mathrm{mL}^{-1}, 0.20 \mathrm{U} \cdot \mathrm{mL}^{-1}$ and $46 \mu \mathrm{M}$ after the first injection, and $1.3 \mathrm{U} \cdot \mathrm{mL}^{-1}, 0.26 \mathrm{U} \cdot \mathrm{mL}^{-1}$ and $35 \mu \mathrm{M}$ after the second injection. Concentrations of enzymes are given assuming that GOx and HRP remained sequestrated in the GUV, whereas AR concentration is given assuming all AR was consumed or has diffused to the outside after the first injection. B. The resorufin production is reported by the mean fluorescence intensity inside the GUV over time detected by LSCM. C. Time-lapse images of a GUV during and after the injection, showing the fluorescence intensity increase followed by its decay Scale bar: $50 \mu \mathrm{m}$. Pseudo-colour, LUT: fire. Average injected volume: $1250 \pm 70 \mathrm{pL}$. Initial GUV diameter: $138 \mu \mathrm{m}$; GUV diameter after 2 injections: $196 \mu \mathrm{m}$.

To complete this systematic study, a final set of experiments was performed. Here, the initial substrates $(50 \mu \mathrm{M}$ glucose and $50 \mu \mathrm{M}$ AR) were encapsulated during the liposome growing step. The enzymes (GOx and HRP) were injected only once per vesicle (table S5, ESI) and repeated on at least ten vesicles and for various concentrations of substrates (see Figure S11). As shown with the typical obtained signal in Figure 7, we observed a regular rise of the resorufin signal inside the vesicle over the first minute. Then, the intensity remained stable for a long time, at least $15 \mathrm{~min}$ and up to 1 hour when monitored (long time experiments were not performed systematically). This plateau corresponds to a balance between the production of resorufin inside the liposome and its diffusion to the outside of the GUV. The steady-state concentration of resorufin was estimated at $\sim 0.5 \mu \mathrm{M}$ based on results from Figure 3 . This mean concentration of produced resorufin, reported by the plateau intensity, was also shown to depend on the AR concentration in the solution (Figure S11). 


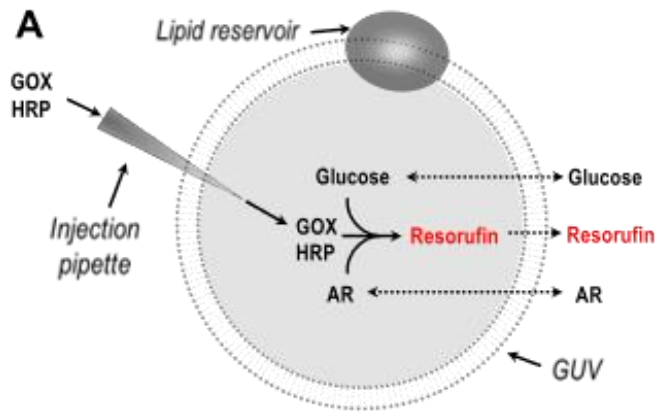

B

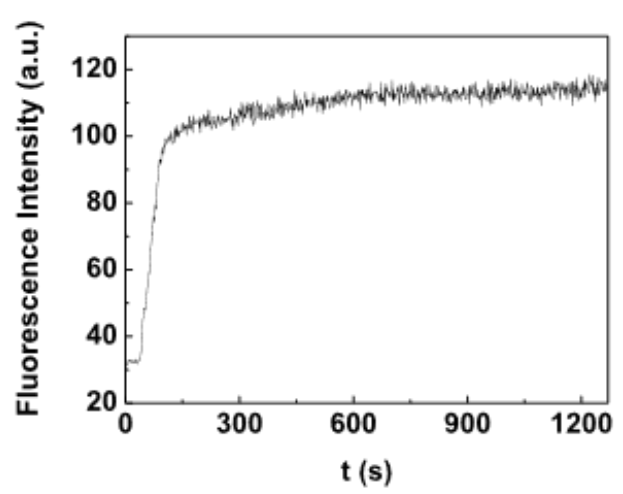

C

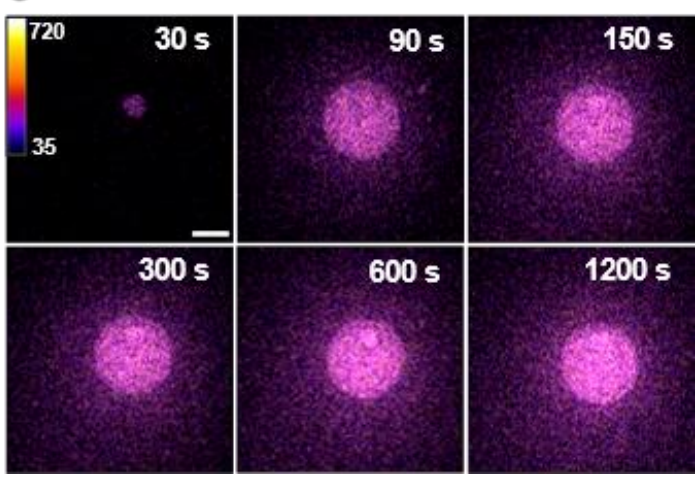

Figure 7. A. Schematic representation of the Amplex Red assay performed in a GUV. A GUV containing glucose $(10 \mu \mathrm{M})$ and $A R(50 \mu \mathrm{M})$ was injected once with a mix of GOx (4.76 U. $\mathrm{mL}^{-1}$ in situ concentration) and HRP (0.38 U. $\mathrm{mL}^{-1}$ in situ concentration). B. The resorufin production is reported by the mean fluorescence intensity inside the GUV over time detected by LSCM. C. Microscopy time-lapse images obtained by LSCM of a GUV during and after injection, showing the fluorescence intensity increase until reaching a steady-state. Scale bar: $50 \mu \mathrm{m}$. Pseudo-colour, LUT: fire. Injected volume: $630 \mathrm{pL}$. Initial GUV diameter: $39 \mu \mathrm{m}$; GUV diameter after injection: $108 \mu \mathrm{m}$.

Finally, we demonstrated that we could manage a long-lasting generation of a compound (resorufin) produced by a bienzymatic reaction inside a single vesicle. Moreover, the observed steady-state response also provides clues on the diffusion of the substrates, glucose and AR across the membrane. We may expect a drop of fluorescence intensity after few min, caused by a substrate runout. However, the signal in the reactor was very stable for long durations (tens of minutes). This proves that glucose and AR substrates can diffuse across the membrane while the enzymes remain sequestrated. Our results confirm previous reports on the diffusion of $\mathrm{AR}^{36}$ across lipid membranes but also show a sufficient permeability of our vesicles to glucose, which allows to perform stably the bienzymatic reaction inside.

Usually, phospholipid bilayers are considered as weakly permeable to glucose (permeation coefficient of $10^{-10} \mathrm{~cm} \cdot \mathrm{s}^{-1}$ ) 37 . However, in the vast majority of reported studies, liposomes are composed of ideal mixtures of one or two types of pure synthetic phospholipids (DPPC, POPC ...). Herein, GUVs were formed from a natural mix (soybean polar extract) composed of phospholipids with a wide variety of polar heads (PC, PE, PI and PA) and fatty acid chains (DO, DP, PO...) (see the Chemicals section), to mimic the complexity of living cell membrane composition. With such a composition, formation of lipid rafts is possible ${ }^{38}$. A mix of fluidic areas where phospholipids have a low transition temperature $T_{m}$ and more rigid phases where $T_{m}$ is higher is expected. These spatial differences on the membrane might favour permeation to small molecules. Moreover, all present experiments were achieved in solutions of physiological osmolarity (with respect to eukaryotic cells) and at low sugar concentrations $(10-100 \mu \mathrm{M})$ whereas usual GUV experiments are performed either in pure water or buffers supplemented with high sugar concentrations (200-300 $\mathrm{mM}$ sucrose). This last condition increases the solution viscosity and therefore slows down diffusion and reaction kinetics. As well, it affects the lipid diffusion in membranes and consequently hampers their permeation to small molecules ${ }^{39,40}$. This additionally explains the semi-permeable behaviour of the current GUVs as compared to other purely synthetic GUVs in other experimental conditions. Overall, this feature is used in the present study as an advantage to let small molecules, i.e. the enzymatic substrates, diffuse from the outer solution towards the internal volume and activate the enzymatic reactions.

Additionally, one may argue that phospholipids could be oxidized and henceforth increase membrane permeability ${ }^{41}$. As we showed in control experiments, neither microinjection nor electric pulses are responsible for the semi-permeability of GUVs (Figures S5 and S6). Additionally, the presence of hydrogen peroxide in some of the experiments may lead to phospholipid peroxidation and consequently could modify the permeability of the liposomes ${ }^{42}$. However, the membrane semi-permeability was similar in presence or absence of hydrogen peroxide (Figures S5 and S6). The formation of transient nanopores is more likely to be the source of the observed semi-permeable feature, although these could not be observed directly in our microscopy experiments.

The result from Figure 7 provides information allowing to clarify the previous results of Figure 5 . First, we can conclude that the slow decay observed on Figure 5 is not likely due to the diffusion of HRP to the outside of the vesicle since we did not observe any fluorescence decay on Figure 7, for a similar experimental time-range. We showed that $A R$ as a cosubstrate of HRP can permeate from the outside to the inside of the vesicle, however certainly slower than $\mathrm{H}_{2} \mathrm{O}_{2}$. Thus, the slow activity decay of Figure 5 may result from a limited diffusion of AR across the membrane, reducing the production rate of resorufin over time. However, under well-chosen conditions of reactant concentrations such as shown in Figure 
7, the Amplex Red assay can effectively be performed with micro-units of enzymes inside a stable nanolitre-volume reactor. Kinetic and quantitative accuracies are similar to bulk experiments but at the scale of an individual liposome.

\section{Conclusions}

The GUVs formed by swelling on a lipid reservoir allow single biomimetic compartment manipulation, injection and monitoring in usual conditions of biochemical analyses (buffers, reactants, concentrations, $\mathrm{pH}$ etc.). We characterized their peculiar permeability features (permeation of small neutral molecules, typically $<300 \mathrm{~g} \cdot \mathrm{mol}^{-1}$ ) and took advantage of these to probe a bi-enzymatic reaction inside single vesicles. We believe that such compartments allowing out-ofequilibrium reactions may be useful to develop artificial celllike systems while avoiding the use of membrane proteins. In addition, the system is fully suitable to implement other analytical methods, such as in situ electrochemical detection by a microelectrode ${ }^{43}$. The direct detection of hydrogen peroxide flux shall provide complementary quantitative information for the analysis of enzymatic activities.

\section{Conflicts of interest}

There are no conflicts to declare.

\section{Acknowledgements}

This work was financially supported by the Agence Nationale de la Recherche (ANR, NOSYNTHCELL project, ANR-RF-201301). The authors wish to thank Raphael Harismendy (Univ. Bordeaux) for preliminary experiments, $\mathrm{Dr}$ Adeline Perro (Bordeaux INP, ISM, UMR 5255, Pessac, France) for her help to prepare the microscopy slides and $\mathrm{Dr}$ Ann-Sofie Cans (Department of Chemistry and Chemical Engineering, Chalmers University of Technology, Gothenburg, Sweden) and her collaborators for discussions and experimental advices at the onset of this project.

\section{Notes and references}

M. J. York-Duran, M. Godoy-Gallardo, C. Labay, A. J.

Urquhart, T. L. Andresen and L. Hosta-Rigau, Colloids Surfaces B Biointerfaces, 2017, 152, 199-213. R. Dimova, Annu. Rev. Biophys., 2019, 48, 93-119.

P. Walde and S. Ichikawa, Biomol. Eng., 2001, 18, 143-177. J. C. Blain and J. W. Szostak, Annu. Rev. Biochem., 2014, 83, 615-640.

5 M. J. Hope, M. B. Bally, L. D. Mayer, A. S. Janoff and P. R. Cullis, Chem. Phys. Lipids, 1986, 40, 89-107.

6 C. H. Huang, Biochemistry, 1969, 8, 344-352.

7 M. J. Hope, M. B. Bally, G. Webb and P. R. Cullis, BBA Biomembr., 1985, 812, 55-65.

8 M. I. Angelova and D. S. Dimitrov, Faraday Discuss. Chem. Soc., 1986, 81, 303-311.
L.-R. Montes, A. Alonso, F. M. Goñi and L. a Bagatolli, Biophys. J., 2007, 93, 3548-3554.

T. Pott, H. Bouvrais and P. Méléard, Chem. Phys. Lipids, 2008, 154, 115-119.

P. Lefrançois, B. Goudeau and S. Arbault, Integr. Biol., 2018, 10, 429-434.

A. Jesorka, N. Stepanyants, H. Zhang, B. Ortmen, B. Hakonen and O. Orwar, Nat. Protoc., 2011, 6, 791-805. A. Moscho, O. Orwar, D. T. Chiu, B. P. Modi and R. N. Zare, Proc. Natl. Acad. Sci. U. S. A., 1996, 93, 11443-7. M. Criado and B. U. Keller, FEBS Lett., 1987, 224, 172-176. N. N. Deng, M. Yelleswarapu and W. T. S. Huck, J. Am. Chem. Soc., 2016, 138, 7584-7591.

T. Robinson, Adv. Biosyst., 2019, 3, 1800318. H. Mutschler, P. Schwille, R. Lipowsky, R. Dimova, S. Herminghaus, T. J. Erb, J. Spatz, T.-Y. D. Tang, A. Hyman, P. Bastiaens, T. Robinson, K. Sundmacher, S. Wegner, T. Vidakovic-Koch, E. Bodenschatz, P. Bieling, J.-C. Baret, K. Landfester, V. Sourjik and P. Dabrock, Angew. Chemie Int. Ed., 2018, 57, 13382-13392.

W. M. Śmigiel, P. Lefrançois and B. Poolman, Emerg. Top. Life Sci., 2019, 3, 445-458.

I. L. Jørgensen, G. C. Kemmer and T. G. Pomorski, Eur. Biophys. J., 2017, 46, 103-119.

V. Noireaux and A. Libchaber, Proc. Natl. Acad. Sci., 2004, 101, 17669-17674.

S. Li, X. Wang, W. Mu and X. Han, Anal. Chem., 2019, 91, 6859-6864.

C. K. Haluska, K. A. Riske, J.-M. Lehn, R. Lipowsky and R. Dimova, Pnas, 2006, 103, 15841-15846.

W. S. Rasband, ImageJ, U. S. National Institutes of Health, Bethesda, Maryland, USA and https://imagej.nih.gov/ij/, 1997-2018.

S. B. Bankar, M. V. Bule, R. S. Singhal and L. Ananthanarayan, Biotechnol. Adv., 2009, 27, 489-501. V. Leskovac, S. Trivić, G. Wohlfahrt, J. Kandrač and D. Peričin, Int. J. Biochem. Cell Biol., 2005, 37, 731-750. M. Zhou, Z. Diwu, N. Panchuk-Voloshina and R. P. Haugland, Anal. Biochem., 1997, 253, 162-168.

S. G. Rhee, T.-S. Chang, W. Jeong and D. Kang, Mol. Cells, 2010, 29, 539-549.

R. B. Liebherr and H. H. Gorris, Molecules, 2014, 19, 14417-14445.

V. Towne, M. Will, B. Oswald and Q. Zhao, Anal. Biochem., 2004, 334, 290-296.

D. Debski, R. Smulik, J. Zielonka, B. Michałowski, M. Jakubowska, K. Debowska, J. Adamus, A. Marcinek, B. Kalyanaraman and A. Sikora, Free Radic. Biol. Med., 2016, 95, 323-332.

N. E. Barlow, G. Bolognesi, S. Haylock, A. J. Flemming, N. J. Brooks, L. M. C. Barter and O. Ces, Sci. Rep., 2017, 1-12. Y. Tamba, H. Ariyama, V. Levadny and M. Yamazaki, 2010, 12018-12026.

M. B. Mustafa, D. L. Tipton, M. D. Barkley, P. S. Russo and F. D. Blum, 1993, 370-378.

M. Kim, W. G. Chen, J. W. Kang, M. J. Glassman, K. Ribbeck and B. D. Olsen, Adv. Mater., 2015, 27, 4207-4212. 
35

36

A. S. Ladokhin, M. E. Selsted and S. H. White, Biophys. J., 1997, 72, 1762-1766.

H. M. Piwonski, M. Goomanovsky, D. Bensimon, A.

Horovitz and G. Haran, Proc. Natl. Acad. Sci., 2012, 14371443.

37 R. E. Wood, F. P. Wirth and H. E. Morgan, Biochemistry, 1968, 63, 171-178.

38 M. Cebecauer, M. Amaro, P. Jurkiewicz, M. J. Sarmento, R. Šachl, L. Cwiklik and M. Hof, Chem. Rev., 2018, 118, 1125911297.

39 A. Roy, R. Dutta, N. Kundu, D. Banik and N. Sarkar, Langmuir, 2016, 32, 5124-5134.

40 G. Van Den Bogaart, N. Hermans, V. Krasnikov, A. H. De Vries and B. Poolman, Biophys. J., 2007, 92, 1598-1605.

41 L. Rems, M. Viano, M. A. Kasimova, D. Miklavčič and M. Tarek, Bioelectrochemistry, 2019, 125, 46-57.

42 J. E. Smolen and S. B. Shohet, J. Lipid Res., 1974, 15, $273-$ 280.

43 2011, 15, 1437-1450. 